\title{
La atención del alumnado con aptitudes sobresalientes por parte de las egresadas de la LEE 2004
}

\author{
Attention of gifted students by the 2004 LEE graduates
}

Pedro Covarrubias Pizarro

Pedro Covarrubias Pizarro. Red de Investigadores Educativos Chihuahua A.C., México. Es doctor en Educación por la UACH, maestro en Educación Especial por la UATx y miembro de la Red de Investigadores Educativos Chihuahua. Actualmente asesor e investigador independiente en el tema de las altas capacidades. Excatedrático de la IByCENECH, ponente y tallerista en diferentes congresos nacionales e internacionales. Asesor de tesis de licenciatura y maestría, dictaminador de ponencias para congresos, así como de artículos arbitrados de diferentes revistas científicas. Autor de artículos arbitrados, ponencias y capítulos de libros. Correo electrónico: pe.covarrubias@gmail.com. ORCID: https://orcid.org/0000-0002-10724462.

\begin{abstract}
Resumen
El presente reporte parcial de investigación corresponde a un proyecto de seguimiento con alumnas y alumnos egresados de las diferentes generaciones que cursaron la licenciatura en Educación Especial, plan de estudios 2004, en la Benemérita y Centenaria Escuela Normal del Estado de Chihuahua. El propósito central gira en torno a identificar las prácticas que han realizado con respecto a la atención de las niñas, niños y jóvenes con aptitudes sobresalientes, ${ }^{1}$ durante sus años de servicio, ya que dentro de la malla curricular del plan de estudios cursaron una asignatura específica denominada "Atención educativa de alumnos con aptitudes sobresalientes". Ante la problemática que se ha detectado en la entidad por la baja población atendida con esta condición, surge el interés de indagar con las egresadas el trabajo que realizan o han realizado en este sentido. En esta primera parte del trabajo de investigación se presentan los resultados obtenidos con la aplicación de una encuesta de corte cuantitativo, y se exponen resultados de tipo descriptivo con algunas interpretaciones e inferencias realizadas a partir de los datos duros. Posteriormente se contempla una segunda parte del proyecto, con un enfoque cualitativo. Uno de los hallazgos encontrados es que la asignatura impartida en la licenciatura fue significativa para el desarrollo profesional del alumnado egresado.
\end{abstract}

Palabras clave: Aptitudes sobresalientes, seguimiento, malla curricular.

\begin{tabular}{l} 
Abstract \\
This partial research report corresponds to a follow-up project with female and male \\
graduates of different generations who completed the Bachelor of Special Educa- \\
tion, 2004 Curriculum, at the Benemérita y Centenaria Escuela Normal del Estado de \\
Chihuahua. The central purpose revolves around identifying the practices they have \\
carried out with gifted students, during their years of service, since within the cur- \\
\hline 1 Para la traducción del concepto de aptitudes sobresalientes se emplea el término gifted \\
students como sinónimo, ya que es la denominación equivalente al término en el \\
idioma inglés.
\end{tabular}


riculum they took a specific subject called "Educational attention of gifted students". Given the problems that have been detected in the entity by the low population served with this condition, the interest arises to inquire with the graduates about the work they do or have done in this regard. In this first part of the research work the results obtained with the application of a quantitative survey are presented, and descriptive results are presented with some interpretations and inferences made from hard data. Subsequently, a second part of the project is contemplated, with a qualitative approach. One of the findings is that the subject taught in the Bachelor's degree was significant for the professional development of the graduated students.

Keywords: Gifted students, follow-up, curriculum mesh.

\section{INTRODUCCIÓN}

El reporte de resultados parciales que se expone en el presente documento corresponde a una investigación de seguimiento de egresadas ${ }^{2}$ que cursaron el plan de estudios 2004 de la Licenciatura en Educación Especial -LEE- (SEP, 2004a) en la Benemérita y Centenaria Escuela Normal del Estado de Chihuahua-IByCENECH-. Como pregunta de investigación se plantea: ¿Cuál es el trabajo que han desarrollado las egresadas de la LEE 2004, con respecto a la atención del alumnado con aptitudes sobresalientes, en sus años de servicio? Aunadas a esta pregunta eje se plantearon las siguientes preguntas de investigación: ¿Cómo valora la asignatura tomada en la licenciatura para dar respuesta a la atención de niñas, niños y jóvenes con aptitudes sobresalientes?, ¿Cuáles son las principales barreras que ha enfrentado en su práctica profesional para poder atender a la población sobresaliente? y ¿Cómo vislumbra el futuro de esta población ante los cambios y retos que conlleva la actual reforma educativa de la Nueva Escuela Mexicana?

En este marco de acción el objetivo particular del proyecto se delimita de la siguiente forma: Analizar el trabajo que han realizados las egresadas de la LEE 2004, con respecto a la atención del alumnado con aptitudes sobresalientes. La conceptualización que realiza la Secretaría de Educación Pública de esta población es que los niños, niñas y jóvenes con aptitudes sobresalientes son

[...] aquellos capaces de destacar significativamente del grupo social y educativo al que pertenece en uno o más de los siguientes campos del quehacer humano: científico-tecnológico, humanísticosocial, artístico o de acción motriz. Estos alumnos, por presentar necesidades específicas, requieren de un contexto facilitador que les permita desarrollar sus capacidades personales y satisfacer sus necesidades para su propio beneficio y el de la sociedad [SEP, 2006, p. 59].

Como parte del desarrollo introductorio es necesario exponer tres antecedentes fundamentales: el origen del plan estudios de la LEE 2004 en la IByCENECH, el

2 Se emplea el término de egresadas únicamente, ya que conforman la mayoría de la población que participó en la investigación, sin que esto omita las pocas encuestas contestadas por algunos varones egresados. 
análisis de la asignatura "Atención educativa de alumnos con aptitudes sobresalientes" que cursaron durante su formación inicial, y finalmente la problemática de atención en educación básica del Estado que presenta el alumnado con aptitudes sobresalientes - AS-.

Con respecto al primer tópico, la IByCENECH solamente había formado normalistas en la licenciatura en Educación Primaria o en Educación Preescolar. La formación en educación especial no se contemplaba en ninguna institución formadora de docentes en el Estado. En el año 2004 se implementó por primera vez el plan de estudios de la LEE, en el área de discapacidad intelectual. Una característica de esta oferta educativa fue que solo se abrió un grupo por generación, por lo tanto el número de egresadas hasta el año 2019 era de 319 alumnas y alumnos. Este plan de estudios dejó de ofertarse en agosto del 2018, para dar paso a la licenciatura en Inclusión Educativa (SEP, 2018b).

Una parte fundamental del planteamiento del problema guarda relación con la asignatura "Atención educativa de alumnos con aptitudes sobresalientes" que cursaron las egresadas, ya que dentro de la malla curricular se incluía la materia ubicada en el sexto semestre, con carga de cuatro horas por semana y siete créditos (SEP, 2004b). El propósito general de la asignatura era "brindar un marco teórico y conceptual, que permita fundamentalmente al estudiante, conocer, explicar y atender la aptitud sobresaliente bajo diversos enfoques teóricos (...) introducirlo en el campo de estudio de los diferentes modelos explicativos y las oportunidades de intervención psicoeducativa" (p. 1).

El tercer punto que permite contextualizar la problemática se desprende del análisis de la estadística que se reporta en la entidad con respecto a las niñas, niños y jóvenes con AS. Esta estadística emana del Formato 911 que emite la Secretaría de Educación Pública -SEP- y que da cuenta de la población que atienden los servicios de Educación Especial-EE-. En la tabla 1 se muestran los resultados que se obtuvieron al respecto.

Tabla 1. Estadística de alumnado con aptitudes sobresalientes en el estado de Chihuahua.

\begin{tabular}{|l|c|c|c|c|c|c|c|c|c|}
\hline \multicolumn{6}{|c|}{ Aptitudes sobresalientes atendidos por USAER en el ciclo 2018-2019 (cifras 911) } \\
\hline Nivel & \multicolumn{3}{|c|}{ Preescolar } & \multicolumn{3}{c|}{ Primaria } & \multicolumn{3}{c|}{ Secundaria } \\
\hline \multirow{3}{*}{$\begin{array}{l}\text { Entidad: } \\
\text { Chihuahua }\end{array}$} & $\mathrm{H}$ & $\mathrm{M}$ & $\mathrm{T}$ & $\mathrm{H}$ & $\mathrm{M}$ & $\mathrm{T}$ & $\mathrm{H}$ & $\mathrm{M}$ & $\mathrm{T}$ \\
\cline { 2 - 9 } & 120 & 125 & $\mathbf{2 4 5}$ & 622 & 500 & $\mathbf{1 1 2 2}$ & 231 & 262 & $\mathbf{4 9 3}$ \\
\hline
\end{tabular}

Fuente: SEP (2019a).

De acuerdo con la información obtenida, en el estado de Chihuahua se tiene reportada una población total de 1,860 alumnos con AS, distribuidos en los tres niveles educativos. Al revisar la estadística estatal de educación básica, la población del ciclo escolar 2018-2019 hace un total de 746,533 alumnos y alumnas (SEP, 2019b). Esto 
indica que solamente el $.24 \%$ de esa población regular ha sido identificada con AS.

De estos datos es de donde surge la inquietud de indagar qué han realizado las egresadas de la IByCENECH que laboran en el nivel de educación especial con respecto a la población del alumnado con AS.

\section{Metodología}

Este reporte de investigación corresponde a un estudio de corte cuantitativo descriptivo. Se rescataron algunos atributos de la población objeto de estudio y se tomaron decisiones considerando que el proyecto se ejecutó en el área educativa. Hernández, Fernández y Baptista (2010) proponen que hacer investigación cuantitativa representa un proceso sistemático de pasos que se aplican al estudio de un fenómeno que determina el objeto de estudio y la selección del diseño de la investigación. Por su parte, Luna (2014) considera que los propósitos en esta área -la educativa-, y bajo un enfoque cuantitativo, siguen pasos secuenciales en los cuales debe haber cierta flexibilidad que permita la redefinición de algunos elementos tanto en los instrumentos como en el análisis de los resultados.

Técnica de investigación

El instrumento que se empleó fue un cuestionario tipo encuesta, que consta de 20 reactivos: seis ítems de datos generales, ocho correspondientes a indagar el trabajo realizado con el alumnado AS en su trayectoria profesional, tres relacionados con la valoración de la asignatura cursada en su formación inicial y tres de opinión personal. La técnica de encuesta es ampliamente utilizada como procedimiento de investigación, ya que permite obtener y elaborar datos de modo rápido y eficaz. Como instrumento para la recolección de datos ofrece la ventaja y posibilidad de llevar a cabo una aplicación masiva y obtener un abanico de posibilidades al plantearse diversos tópicos (Casas, Repullo y Donado, 2003).

El instrumento fue elaborado por medio del formulario académico de Google, ya que esto permitió la rápida difusión y llenado por parte de las egresadas de la LEE 2004, quienes formaron una red de comunicación. Se obtuvo el consentimiento de las participantes y se consideraron los criterios de privacidad y confidencialidad, para cumplir con los requerimientos éticos de la investigación. La participación se dio de manera voluntaria y con consentimiento informado (González, 2002).

Previo al envío del formulario fue sometido a la prueba de fiabilidad para determinar la consistencia interna, basado en el alfa de Cronbach por medio del programa estadístico SPSS. De acuerdo con los resultados, se obtuvo una confiabilidad de .845 y, considerando que las medidas esperadas deben superar el .70, el coeficiente obtenido le brinda fiabilidad al instrumento diseñado (Oviedo y Campos-Arias, 2005). 


\section{Población participante}

La población destino de la investigación estuvo conformada por las egresadas de la LEE 2004 de la IByCENECH. A partir del año 2008 hasta el 2019 se han licenciado doce generaciones. Según los registros estadísticos de la institución proporcionados por el Departamento de Control Escolar, la población total de egresados es de 319 licenciados(as) en Educación Especial. De esta población se obtuvo una respuesta de 123 participantes, lo cual representa el 38.5\% de la población, porcentaje que le da representatividad a los resultados.

En la tabla 2 se muestran algunos de los datos generales de esta población participante.

Tabla 2. Estadística de datos generales de las participantes.

\begin{tabular}{|c|c|c|c|c|c|c|c|c|c|c|c|c|c|c|}
\hline 1. Generación & 2008 & 2009 & 2010 & 2011 & & 012 & 2013 & 2014 & 2015 & 2010 & 2017 & \multicolumn{2}{|c|}{2018} & 2019 \\
\hline$\%$ participantes & $24 \%$ & $6 \%$ & $5 \%$ & $4 \%$ & & $7 \%$ & $3 \%$ & $2 \%$ & $6 \%$ & $11 \%$ & $11 \%$ & 10 & & $5 \%$ \\
\hline \multicolumn{3}{|c|}{ 2. Servicio en que labora ${ }^{3}$} & \multicolumn{2}{|c|}{ USAER } & \multicolumn{2}{|c|}{ CAM } & \multicolumn{2}{|c|}{ Ambos } & \multicolumn{2}{|c|}{ A. Hospital } & \multicolumn{2}{|c|}{ Fuera de EE } & \multicolumn{2}{|c|}{ Particular } \\
\hline \multicolumn{3}{|c|}{$\%$ de respuestas } & \multicolumn{2}{|c|}{$76 \%$} & \multicolumn{2}{|c|}{$16 \%$} & \multicolumn{2}{|c|}{$2 \%$} & \multicolumn{2}{|l|}{$1 \%$} & \multicolumn{2}{|l|}{$4 \%$} & & $\%$ \\
\hline \multicolumn{3}{|c|}{ 3. Función que desempeña ${ }^{4}$} & \multicolumn{3}{|c|}{ M. A. USAER } & \multicolumn{3}{|c|}{ M. A. CAM } & \multicolumn{3}{|c|}{ ctivo(a) } & \multicolumn{3}{|c|}{ Otra función } \\
\hline \multicolumn{3}{|c|}{$\%$ de respuestas } & \multicolumn{3}{|c|}{$70 \%$} & \multicolumn{3}{|c|}{$9 \%$} & \multicolumn{3}{|c|}{$6 \%$} & \multicolumn{3}{|c|}{$15 \%$} \\
\hline
\end{tabular}

Fuente: Construcción personal con base en los datos de SPSS.

Con los datos generales que se obtuvieron de las participantes, se puede considerar que la mayoría de ellas han estado trabajando como maestras de apoyo en los servicios de USAER, por lo tanto, la experiencia de trabajar con alumnado sobresaliente podría ser alta, ya que los Servicios de Educación Especial -USAER-, de acuerdo con la normativa nacional, "son las instancias que están a disposición de la comunidad para apoyar la atención escolar de los alumnos que por alguna condición de discapacidad, aptitudes sobresalientes (...), enfrentan barreras para el aprendizaje y la participación..." (SEP, 2018a, p. 9).

También se rescata como dato importante que el $67 \%$ de las participantes, cuenta con una antigüedad que va de los cuatro a los once años de servicio, lo cual permite suponer que se ha tenido la experiencia suficiente en el campo de trabajo para atender a la población con AS.

\section{Resultados}

Para llevar a cabo el análisis de los resultados se agruparon en tres categorías, mismas en las que contemplan los hallazgos más relevantes de la investigación.

3 USAER = Unidad de Servicio de Apoyo a la Educación Regular, CAM = Centro de Atención Múltiple, AH = Aula Hospitalaria, $\mathrm{EE}=$ Educación Especial.

4 M.A. = Maestra(o) de Apoyo. 
Atención del alumnado con aptitudes sobresalientes

En este apartado se indagó en primer momento si durante su trayectoria profesional habían atendido niñas, niños o jóvenes con aptitudes sobresalientes, a lo cual se obtuvo una respuesta favorable del 84.4\% que contestó que sí. El 15.6\% que dijo no haber trabajado con esta población es correspondiente a aquellas egresadas que laboran en los Centros de Atención Múltiple (16\%) o que trabajan fuera del nivel de educación especial (15\%). Un dato importante que se rescató es que el 34.4\% de las participantes manifestó que durante toda su trayectoria profesional han atendido alumnado AS, y el 45.2\% manifestó haberlo hecho en algunos años de su trayecto. Esto se puede explicar porque las participantes han transitado de servicios a lo largo de su vida laboral, pueden haber trabajado en CAM o en USAER -en donde no siempre se atiende a esta población- y después haber cambiado de modalidad.

En la tabla 3 se muestran algunas de las respuestas que emitieron las egresadas al preguntarles sobre los motivos por los cuales consideran que no se ha trabajado de manera permanente con esta población.

Tabla 3. Motivos por los cuales no se ha trabajado de manera permanente con la población sobresaliente.

\begin{tabular}{|l|l|}
\hline$\%$ & Respuestas seleccionadas \\
\hline $20 \%$ & Trabajo exclusivamente en CAM \\
\hline $30 \%$ & La USAER en la que laboro no le da prioridad a las y los sobresalientes \\
\hline $30 \%$ & No hay población con aptitudes sobresalientes en las escuelas en donde trabajo \\
\hline $20 \%$ & Otras respuestas variadas \\
\hline
\end{tabular}

Fuente: Construcción personal con base en los datos de SPSS.

Esta tabla resulta relevante por dos motivos, el primero de ellos porque de alguna manera explica la falta de población atendida por parte de los servicios de Educación Especial en el Estado, al no considerarlos como prioritarios. Y por otro lado, porque se pone en evidencia uno de los principales mitos que rodean a esta población y que permea en muchos docentes, al considerar que no hay alumnado con estas características en determinados centros escolares o contextos, sobre todo cuando son de zonas vulnerables socioeconómica y culturalmente hablando. Como ya se mencionó, la Propuesta de intervención emitida por la SEP para atender a esta población determina que un alumno(a) con AS es aquel "que destaca significativamente del grupo social y educativo al que pertenece" (SEP, 2006, p. 59), con lo cual se ratifica que en todos los centros escolares y contextos se pueden identificar este tipo de alumnos.

\section{Formación inicial y actualización}

En esta categoría de análisis se exploró el referente que se tiene con respecto a la asignatura cursada en la LEE 2004, en la cual se aborda la atención educativa del alumnado con AS. El cuestionamiento giró en torno a cómo valoraban el curso tomado 
en el sexto semestre con respecto a su vida profesional y la atención de la población en cuestión. Las respuestas que emitieron se expresan en la tabla 4.

Tabla 4. Valoración del curso de aptitudes sobresalientes que estudiaron en la LEE 2004.

\begin{tabular}{|l|l|}
\hline$\%$ & Respuestas seleccionadas \\
\hline $74 \%$ & Útil y oportuno para mi formación profesional \\
\hline $20 \%$ & Poco productivo para mi formación profesional \\
\hline $0 \%$ & Nada útil en mi formación profesional \\
\hline $6 \%$ & No recuerdo el curso \\
\hline
\end{tabular}

Fuente: Construcción personal con base en los datos de SPSS.

Para la mayoría de las egresadas -74\% - la asignatura resultó ser significativa en su formación inicial y por tanto de utilidad en su carrera profesional, ya que el curso ofrece una visión general de la población con aptitudes sobresalientes, los modelos teóricos que dan sustento al tema, los proceso de detección, evaluación e intervención psicoeducativa (SEP, 2004b). Esto podría ser un referente a considerar al momento de atender o no a esta población, ya que al contar con una formación previa, el trabajo en la práctica no resulta desconocido.

Dentro de esta categoría se indagó el nivel de conocimiento que tienen las egresadas con respecto a la Propuesta de intervención de la SEP (2006) - la cual se analizó en el currículo de la asignatura-, que rige la operatividad estipulada en la normativa nacional para atender a esta población dentro de los servicios de EE. En la figura 1 se presentan las cuatro preguntas que comprende este rubro.

De manera general se puede apreciar que la mayoría de las egresadas conocen la Propuesta de Intervención de la SEP (2006), en donde se especifican los mecanismos para llevar a cabo el proceso de detección inicial, el proceso de evaluación psicopedagógica y los tres tipos de enriquecimiento -áulico, escolar y extracurricular- que se proponen para atender en las escuelas regulares al alumnado con AS. La mayor área de oportunidad se encuentra en la realización de la evaluación psicopedagógica, ya que al ser cinco tipos de aptitudes las que pueden identificarse -intelectual, creativa, socioafectiva, psicomotriz y artística-, cada una de ellas implica procesos e instrumentos diferentes. En este rubro, el 40\% de las participantes contestaron que no lo conocen o lo conocen muy poco. Nuevamente hay que recordar que una parte de la egresadas laboran en los CAM o tienen otras funciones, y eso explicaría el motivo por el cual desconocen la Propuesta y sus diferentes etapas.

Las egresadas participantes en la investigación también fueron cuestionadas con respeto a la capacitación que han tomado en su trayectoria profesional para atender a la población con AS. En este sentido, solo el 13\% contestó que lo ha hecho frecuentemente, el 60\% manifestó haberse capacitado solo ocasionalmente, y el 27\% expresó que nunca ha tomado algún curso al respecto. Estos datos permiten entender algunas de las respuestas ya expuestas, ya que ante la ausencia de mecanismos o procesos de 


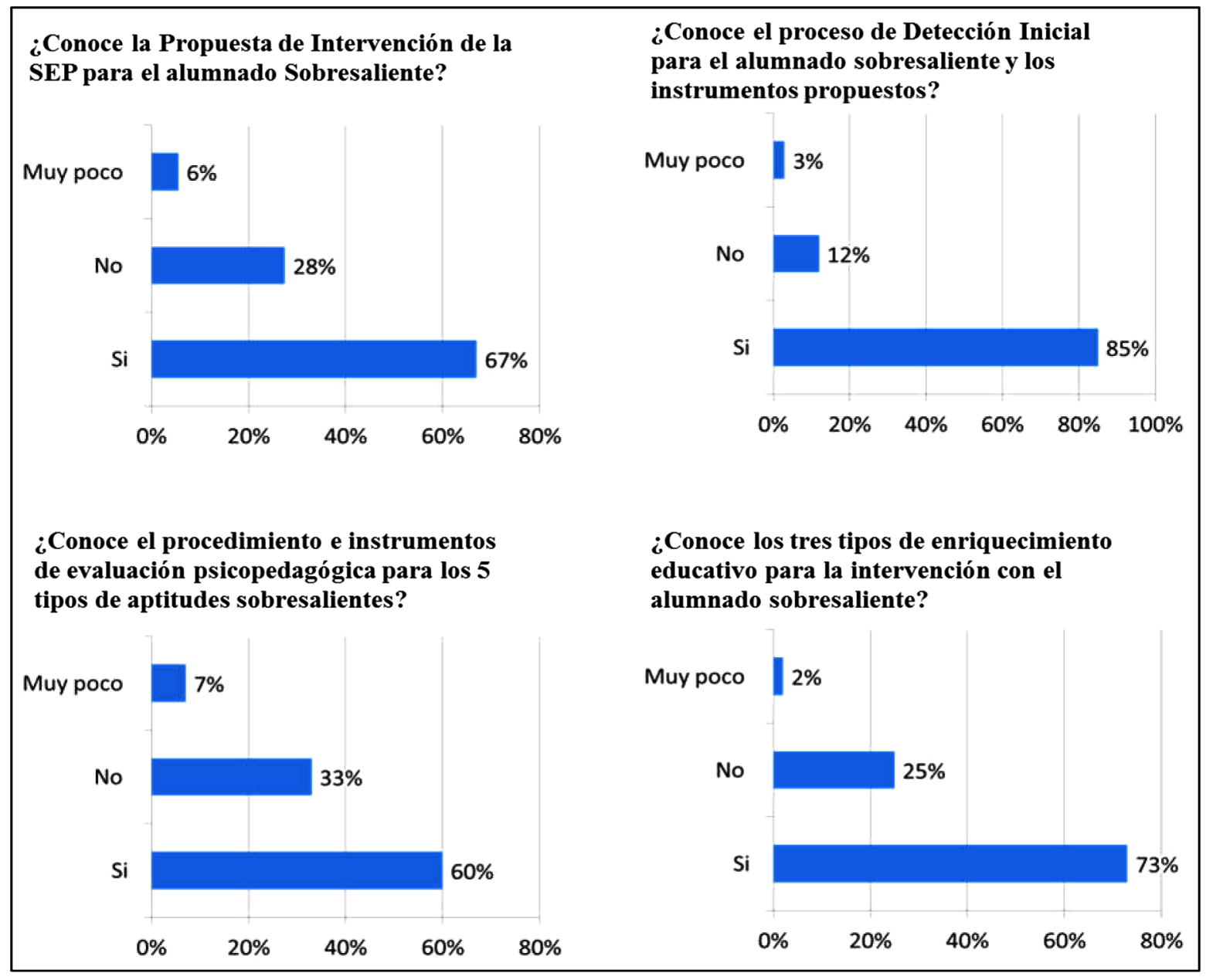

Figura 1. Conocimiento de los procedimientos de detección, evaluación e intervención. Fuente: Construcción personal con base en los datos de SPSS.

capitación, la tarea de identificar, evaluar y atender al alumnado con AS se vuelve un proceso complejo o incluso desmotivador.

\section{Expectativas y creencias}

La última categoría de análisis agrupa una serie de preguntas que guardan relación con ciertas expectativas y creencias con respecto a la atención educativa de la población con AS. Una de las preguntas fundamentales fue para indagar si las egresadas consideraban si la escuela regular y los servicios de Educación Especial estaban en condiciones de atender al alumnado sobresaliente. En este sentido, se puso en evidencia que aún permea una baja expectativa al respecto, ya que el 70\% de las participantes dijo que faltaban muchas condiciones en las escuelas regulares para lograrlo, y el 69\% también dijo que faltaban muchas condiciones en los servicios de Educación Especial. Este panorama permite entender la posible causa de la baja estadística de alumnos atendidos en la entidad, ya que si se percibe como un hecho complicado brindar la atención educativa, entonces eso determina el actuar de las maestras de apoyo. 
Al indagar los principales obstáculos que identifican para atender a esta población en los servicios de Educación Especial y en las escuelas regulares, las respuestas emitidas fueron las que se muestran en la tabla 5.

Tabla 5. Obstáculos para atender a la población sobresaliente.

\begin{tabular}{|l|l|}
\hline$\%$ & Respuestas seleccionadas \\
\hline $51 \%$ & Prevalece el interés por atender a la discapacidad \\
\hline $20 \%$ & La desvinculación entre la escuela regular y el servicio de educación especial \\
\hline $17 \%$ & La actitud de las y los docentes tanto de la escuela regular como de los servicios de EE \\
\hline $5 \%$ & Falta de recurso materiales para apoyar a estos alumnos \\
\hline $5 \%$ & Desconocimiento del tema \\
\hline $2 \%$ & Otras respuestas \\
\hline
\end{tabular}

Fuente: Construcción personal con base en los datos de SPSS.

Estas respuestas pueden considerarse congruentes con las opiniones antes revisadas, ya que podrían explicar, entre otras cosas, los motivos de la no-atención y la visión de que faltan muchas condiciones para lograr una adecuada intervención. Finalmente, también se indagó qué futuro le espera a la población sobresaliente ante la Nueva Escuela Mexicana y los cambios que se han generado tanto en el artículo $3^{\circ}$ constitucional (DOF, 2019) como en la Ley General de Educación (Presidencia de la República, 2019), en donde se enmarca la Educación Inclusiva como principio educativo y se sigue contemplando dentro de la diversidad al alumnado con AS. En este sentido, solo el 21\% de las participantes considera que esta población tendrá un nuevo impulso en la política educativa, el 33\% opina que seguirá siendo una población no atendida, y el $42 \%$ manifiesta un futuro incierto para ellos.

\section{CONCLUSIONES}

Como conclusiones generales se pueden inferir los siguientes datos que dan respuesta a las preguntas de investigación:

1. Las alumnas egresadas consideran que la formación que obtuvieron al cursar la LEE 2004 y en particular la asignatura que aborda la atención educativa para el alumnado con AS les permitió adquirir herramientas necesarias para dar respuesta a esta población que se encuentran en los servicios de Educación Especial, por lo tanto el curso que tomaron cumple con los propósitos para los cuales fue diseñado.

2. Las exalumnas han atendido a la población AS en la medida en que los servicios lo han permitido, ya que en varios servicios de USAER no se le ha dado prioridad a este alumnado, o bien laboran en los CAM, en donde no se atienden las aptitudes sobresalientes o la doble excepcionalidad -discapacidad y AS-.

3. La baja estadística que se reporta en la entidad de población con AS podría explicarse porque no solo depende de las exalumnas formadas en este campo, ya que 
si se considera que actualmente existen 1,057 docentes de apoyo que laboran en USAER en ambos subsistemas, en todo el estado (SEECH, 2019), las egresadas solo representan el 30\% de esta población.

4. La problemática que enfrenta la población con AS en el nivel de educación básica para ser atendida responde a cuestiones multifactoriales que van desde la falta de capacitación y actualización docente, la ausencia de mecanismos de identificación y evaluación por parte de los servicios de EE, el bajo porcentaje del personal que cubre con el perfil de licenciado(a) en Educación Especial, pero sobre todo por la falta de una motivación, sensibilización e interés por esta población, que se vive al interior del sistema educativo estatal y nacional.

\section{REFERENCIAS}

Casas, A. J., Repullo, L. J., y Donado, C. J. (2003). La encuesta como técnica de investigación. Elaboración de cuestionarios y tratamiento estadístico de los datos. Investigación, 527-538.

DOF [Diario Oficial de la Federación] (2019, 15 may.). Decreto por el que se reforman, adicionan y derogan diversas disposiciones de los artículos 3o., 31 y 73 de la Constitución Política de los Estados Unidos Mexicanos. Recuperado de: https://www.dof.gob.mx/ nota_detalle.php?codigo $=5560457 \&$ fecha $=15 / 05 / 2019 \&$ print $=$ true.

González, A. M. (2002). Aspectos éticos en la investigación cualitativa. Revista Iberoamericana de Educación, 85-103.

Hernández, S. R., Fernández, C. C., y Baptista, L. P. (2010). Metodología de la investigación. Perú: McGraw-Hill.

Luna, M. A. (2014). Las variables, su conocimiento y su determinación en el diseño y construcción de instrumentos en métodos cuantitativos. En Á. Díaz Barriga y M. A. Luna, Metodologia de la investigación educativa (pp. 109-139). México: Díaz de Santos.

Oviedo, H. C., y Campos-Arias, A. (2005). Aproximación al uso del coeficiente alfa de Cronbach. Revista Colombiana de Psiquiatría, 572-580.

Presidencia de la República (2019, 30 sep.). Decreto por el que se expide la Ley General de Educación y se abroga la Ley General de la Infraestructura Física Educativa. Recuperado de: http:/ /www. diputados.gob.mx/LeyesBiblio/pdf/LGE_300919.pdf.

SEECH [Servicios Educativos del Estado de Chihuahua] (2019). Estadística de inicio 2019-2020 por sostenimiento. Recuperado de: http://seech.gob.mx/estadistica/descarga/resumenes/ Resumen_I2019.pdf?qt=1588274571222.

SEP [Secretaría de Educación Pública] (2004a). Licenciatura en Educación Especial. Plan de Estudios 2004. México: Secretaría de Educación Pública.

SEP (2004b). Atención educativa a alumnos con aptitudes sobresalientes. México: Secretaría de Educación Pública.

SEP (2006). Propuesta de intervención: atención educativa a alumnas y alumnos con aptitudes sobresalientes. México: Secretaría de Educación Pública.

SEP (2018a). Glosario de términos educativos. México. Recuperado de: http:/ /www.controlescolar. sep.gob.mx/work/models/controlescolar/Resource/307/16/images/normas_controlescolar_basica_16082018.pdf.

SEP (2018b). Dirección General de Educación Superiorpara Profesionales de la Educación. Recuperado de: https://www.cevie-dgespe.com/index.php/planes-de-estudios-2018/51. 
SEP (2019a). Dirección General de Planeación y Programación Estadística Educativa. Recuperado de: http://www.f911.sep.gob.mx/2018-2019/Login.aspx.

SEP (2019b). Dirección General de Planeación, Programación y Estadística Educativa. Recuperado de: http://snie.sep.gob.mx/descargas/estadistica_e_indicadores/estadistica_e_indicadores_educativos_08CHIH.pdf.

Cómo citar este artículo:

Covarrubias Pizarro, P. (2020). La atención del alumnado con aptitudes sobresalientes por parte de las egresadas de la LEE 2004. Revista Electrónica Científica de Investigación Educativa, 5(1), pp. 107-1 17 . doi: doi. org/10.33010/recie.v5i1.991. 